02

\title{
Влияние одноосных давлений на инфракрасные спектры кристаллов $\mathrm{LiNH}_{4} \mathrm{SO}_{4}$
}

\author{
(C) В.И. Стадник ${ }^{1}$, М.Я. Рудиш ${ }^{1,2}$, П.А. Щепанский ${ }^{1,2}$, И.М. Матвиишин ${ }^{1}$, В.М. Габа ${ }^{3}$, О.М. Горина ${ }^{3}$ \\ 1 Львовский национальный университет им. Ивана Франко, \\ 79005 Львов, Украина \\ ${ }^{2}$ Академия им. Яна Длугоша в Ченстохове, \\ 42200 Ченстохова, Польша \\ ${ }^{3}$ Национальный университет „Львовская политехника“, \\ 79013 Львов, Украина, \\ e-mail: vasylstadnyk@ukr.net
}

Поступила в редакцию 03.10.2017 г.

Впервые исследованы инфракрасные спектры отражения механически свободного и зажатого одноосными давлениями кристалла $\mathrm{LiNH}_{4} \mathrm{SO}_{4}$ в спектральной области $600-2000 \mathrm{~cm}^{-1}$ вдоль трех кристаллофизических направлений. С помощью дисперсионных соотношений Крамерса-Кронига получены дисперсионные и барические зависимости оптических постоянных: показателя преломления $n$, действительной $\varepsilon_{1}$ и мнимой $\varepsilon_{2}$ частей диэлектрической постоянной, а также частот продольных $\omega_{\mathrm{LO}^{-}}$и поперечных $\omega_{\mathrm{TO}}$-колебаний, константа затухания $\gamma$ и сила осциллятора $f$ механически свободного и зажатого кристалла $\mathrm{LiNH}_{4} \mathrm{SO}_{4}$. Обнаружено значительное барическое изменение главных полос отражения, что обусловлено влиянием одноосных давлений на тетраэдрические остовы $\mathrm{NH}_{4}$ и $\mathrm{SO}_{4}$.

DOI: $10.21883 /$ OS.2018.02.45527.214-17

\section{Введение}

Кристаллы литий-аммоний сульфата $\mathrm{LiNH}_{4} \mathrm{SO}_{4}$ (ЛАС) - это классические ферроики, которые могут существовать в двух модификациях $[1,2]$. Для $\beta$-модификации кристалла ЛАС характерна псевдогексагональная тридимитоподобная структура, которая состоит из тетраэдров $\mathrm{SO}_{4}$ и $\mathrm{LiO}_{4}$, соединенных вершинами и образующих шестичленные кольца, перпендикулярные к оси $Z$. Половина тетраэдров развернута вершинами вниз, а другая - вверх, где они соединяются с последующим шаром тетраэдров. В образованных полостях находится аммонийная группа $\mathrm{NH}_{4}$. При комнатной температуре элементарная ячейка имеет следующие параметры: $a=5.280 \AA, b=9.140 \AA, c=8.786 \AA[3]$.

Кристаллы ЛАС $\alpha$-модификации отличаются от $\beta$-модификации тем, что тетраэдры $\mathrm{SO}_{4}$ и $\mathrm{LiO}_{4}$ в $Z$-направлении могут иметь не только общие вершины, но и общие ребра. Соседние шары взаимодействуют через водородные связи с атомами азота аммонийных групп, образуя слоистую структуру с орторомбической пространственной группой симметрии Рса2 $2_{1}$ и параметрами решетки $(T=298 \mathrm{~K}) \quad a=10.196 \AA$, $b=4.991 \AA, c=17.100 \AA, V=870.2 \AA^{3}, Z=8$ [4]. Кристаллы $\alpha$-модификации образуются при выращивании при комнатной и более низких температурах, тогда как $\beta$-модификации - при температурах выше $30^{\circ} \mathrm{C}$ [5].

Расчет зонно-энергетической структуры кристаллов ЛАС с использованием плосковолнового псевдопотенциального метода позволил определить ширину запрещенной зоны $E_{g}=5.3 \mathrm{eV}$ [6]. Установлено, что валентные уровни образуются совокупностью узких зон, разделен- ных запрещенными промежутками. Все уровни имеют незначительную дисперсию, что может быть связано с относительно слабым взаимодействием между структурными элементами кристалла $\left(\mathrm{NH}_{4}-\right.$ и $\mathrm{SO}_{4}$-комплексы). Дно зоны проводимости также характеризуется слабой дисперсией в $\boldsymbol{k}$-пространстве и находится в точке Г зоны Бриллюэна. Авторами высказано предположение, что фундаментальный край поглощения связан с прямыми переходами между вершиной валентной зоны и дном зоны проводимости в точке Г центра зоны Бриллюэна.

Кристаллы ЛАС имеют изотропную точку, вследствие чего при длине волны $\lambda=633 \mathrm{~nm}$ и температуре $T=300 \mathrm{~K}$ двупреломление в направлении биссектрисы угла между оптическими осями равно нулю: $\Delta n_{y}=0$ [7].

Исследования кристаллов ЛАС в далекой ИК (инфракрасной) области спектра при комнатной температуpe [8-11] дали возможность определить трансляционные и либрационные моды групп $\mathrm{SO}_{4}^{2-}$ и $\mathrm{NH}_{4}^{+}$и ионов $\mathrm{Li}^{+}$. Определено, что динамика решетки кристаллов $\beta$-ЛАС характеризуется 96 ИК-активными модами. Тетраэдрические группы сульфата и аммония можно рассматривать как молекулярные единицы, которые сравнительно слабо связаны с решеткой. Из общего количества центральных мод 54 являются внутренними модами, которые принадлежат ионам сульфата и аммония, а остальные внешние вращательные и трансляционные оптические моды. Температурные изменения ИК спектров $\alpha$-ЛАС показали, что нагревание кристаллов приводит к постепенному исчезновению внешних мод, которые характеризуют колебательные движения групп $\mathrm{SO}_{4}^{2-}$ и $\mathrm{NH}_{4}^{+}$.

Несмотря на это, исследования влияния внешних полей на ИК спектры кристаллов ЛАС не проводились. 
Ранее исследовалось влияние одноосного давления на спектральные и температурные зависимости показателей преломления ряда изоморфных к ЛАС кристаллов, которые дали возможность установить значительную барическую чувствительность электронной подсистемы этих кристаллов, что проявлялось в значительном смещении по энергетическому спектру положения эффективных полос УФ и ИК осцилляторов [12-14].

Поэтому интересно исследование действия одноосных давлений на ИК спектры с целью выяснения их избирательного влияния на динамику и пространственную ориентацию отдельных структурных элементов кристаллов ЛАС.

\section{Методика эксперимента}

Исследование влияния одноосных давлений на ИК спектры кристаллов ЛАС проводилось с помощью автоматизированного призменного спектрофотометра UR-20, оснащенного специальными приспособлениями для приложения одноосного давления. Прибор UR-20 дает возможность проводить измерения в диапазоне $400-5000 \mathrm{~cm}^{-1}(25-2 \mu \mathrm{m})$.

Кристаллы ЛАС выращивались из водного раствора методом понижения температуры. Исходным сырьем для кристаллов были соединения $\mathrm{Li}_{2} \mathrm{SO}_{4}$ и $\left(\mathrm{NH}_{4}\right)_{2} \mathrm{SO}_{4}$. Во время смешивания исходных компонент в стехиометрическом соотношении получали необходимый раствор соли:

$$
\mathrm{Li}_{2} \mathrm{SO}_{4}+\left(\mathrm{NH}_{4}\right)_{2} \mathrm{SO}_{4} \rightarrow 2 \mathrm{LiNH}_{4} \mathrm{SO}_{4} .
$$

Ориентация образцов проводилась по виду коноскопических фигур и с помощью поляризационного микроскопа.

\section{Результаты и их обсуждение}

На рис. 1 представлены ИК спектры кристаллов $\mathrm{LiNH}_{4} \mathrm{SO}_{4} \alpha$-модификации при комнатной температуре в спектральном диапазоне $600-2000 \mathrm{~cm}^{-1}$ для трех направлений отражения света. В данном диапазоне обнаружены две четкие полосы с максимумами при 1210-1214 и $1455-1476 \mathrm{~cm}^{-1}$, которые обладают значительной дисперсией (табл. 1).

Согласно теории групп, свободный радикал тетраэдрической структуры имеет симметрию $T_{d}$ и 9 внутренних

Таблица 1. Барическое изменение положения полос отражения $\left(\mathrm{cm}^{-1}\right)$ кристалла $\mathrm{LiNH}_{4} \mathrm{SO}_{4}$ при комнатной температуре

\begin{tabular}{c|c|c|c|c}
\hline \multirow{2}{*}{$\begin{array}{c}\text { Кристаллофизическое } \\
\text { направление }\end{array}$} & \multicolumn{2}{|c|}{$v_{3}, \mathrm{~cm}^{-1}$} & \multicolumn{2}{c}{$v_{4}, \mathrm{~cm}^{-1}$} \\
\cline { 2 - 5 } & $\sigma=0$ & $\sigma=\sigma_{m}$ & $\sigma=0$ & $\sigma=\sigma_{m}$ \\
\hline $\mathrm{X}$ & 1214 & 1200 & 1476 & 1479 \\
$\mathrm{Y}$ & 1210 & 1187 & 1455 & 1454 \\
$\mathrm{Z}$ & 1213 & 1196 & 1456 & 1462
\end{tabular}

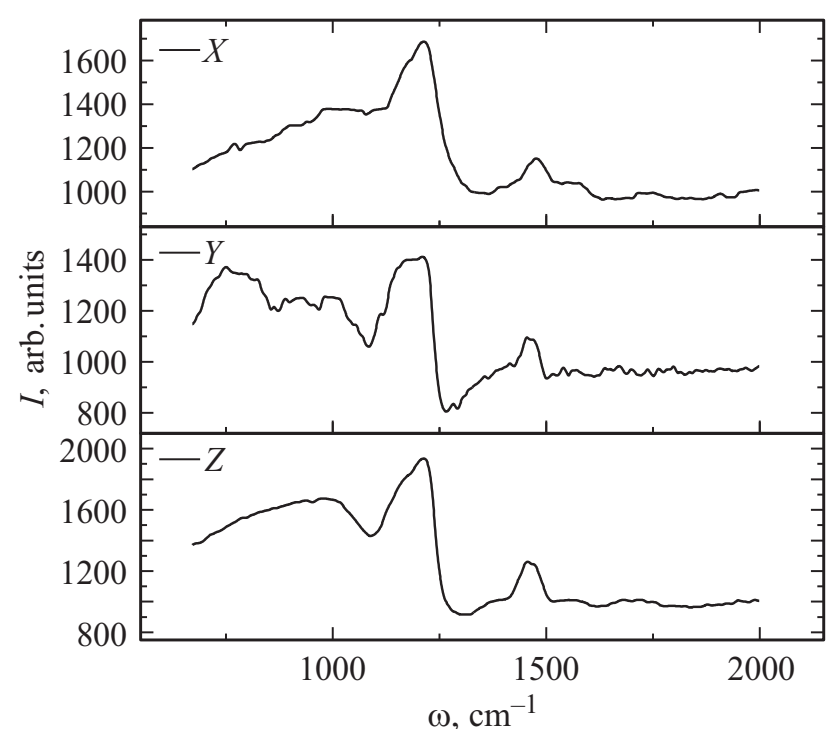

Рис. 1. ИК спектры отражения кристалла $\mathrm{LiNH}_{4} \mathrm{SO}_{4}$ при комнатной температуре для $X$-, $Y$ - и $Z$-направлений.

мод: одну продольную $\left(v_{1}\right)$, двойную поперечную $\left(v_{2}\right)$, тройную продольную $\left(v_{3}\right)$ и тройную поперечную $\left(v_{4}\right)$, среди которых только $v_{3}$ и $v_{4}$ являются активными в ИК области [15]. Моды $v_{1}$ и $v_{2}$ в кристалле расщепляются на невырожденные моды и становятся также активными в ИК спектрах поглощения. Мода $v_{3}=1210-1214 \mathrm{~cm}^{-1}$ соответствует колебаниям тетраэдра $\mathrm{SO}_{4}$, а мода $v_{4}=1455-1476 \mathrm{~cm}^{-1}-$ колебаниям тетраэдра $\mathrm{NH}_{4}$.

Исследованы ИК спектры отражения кристалла $\mathrm{LiNH}_{4} \mathrm{SO}_{4}$ при комнатной температуре при разных значениях одноосного сжатия (рис. 2-4).

Установлено, что одноосное сжатие вдоль основных кристаллофизических направлений приводит к значительным изменениям ИК спектров отражения кристалла $\mathrm{LiNH}_{4} \mathrm{SO}_{4}$.

Так, для спектров отражения в $X$-направлении кристалла $\mathrm{LiNH}_{4} \mathrm{SO}_{4}$ при комнатной температуре наблюдается смещение полосы $v_{3}$ на $14 \mathrm{~cm}^{-1}$ в сторону меньших значений энергий, а полосы $v_{4}-$ на $3 \mathrm{~cm}^{-1}$ в сторону больших энергий при одноосном сжатии вдоль оси $Z$. Для спектров отражения в $Y$-направлении полосы смещаются в низкоэнергетическую сторону: $v_{3} \mathrm{Ha} 23 \mathrm{~cm}^{-1}$, а $v_{4}$ - на $1 \mathrm{~cm}^{-1}$.

Для спектров отражения в $Z$-направлении полоса $v_{3}$ смещается на $17 \mathrm{~cm}^{-1}$ в низкоэнергетическую, а полоса $v_{4}$ - на $6 \mathrm{~cm}^{-1}$ в высокоэнергетическую сторону при комнатной температуре для разных значений одноосного сжатия вдоль оси $Y$.

При сравнении полученных результатов для кристалла ЛАС с результатами для изоморфного кристалла $\left(\mathrm{NH}_{4}\right)_{2} \mathrm{SO}_{4}$ [16], очевидно смещение максимумов в сторону больших энергий. Так, для спектров отражения в $X$-направлении кристалла $\mathrm{LiNH}_{4} \mathrm{SO}_{4}$ при комнатной температуре наблюдается смещение полосы $v_{3}$ на $50 \mathrm{~cm}^{-1}$, 
а полосы $v_{4}-$ на $15 \mathrm{~cm}^{-1}$ в сторону больших энергий по сравнению с аналогичными значениями для кристалла $\left(\mathrm{NH}_{4}\right)_{2} \mathrm{SO}_{4}$. Для спектров отражения в $Y$-направлении полоса $v_{3}$ смещается на $50 \mathrm{~cm}^{-1}$, а полоса $v_{4}-$ на $9 \mathrm{~cm}^{-1}$ в сторону больших энергий. Для спектров отражения в $Z$-направлении полоса $v_{3}$ смещается на $58 \mathrm{~cm}^{-1}$, а полоса $v_{4}-$ на $11 \mathrm{~cm}^{-1}$ также в сторону больших энергий.

Обнаружено значительное отличие в смещении максимумов ИК спектров отражения кристаллов $\left(\mathrm{NH}_{4}\right)_{2} \mathrm{SO}_{4}$ и $\mathrm{LiNH}_{4} \mathrm{SO}_{4}$ при одноосном сжатии. Положения максимумов полос отражения $v_{3}$ и $v_{4}$ в $X$-направлении кристалла $\left(\mathrm{NH}_{4}\right)_{2} \mathrm{SO}_{4}$ смещаются соответственно на 5 и $4 \mathrm{~cm}^{-1}$ в длинноволновую область спектра. Для кристалла $\mathrm{LiNH}_{4} \mathrm{SO}_{4}$ положение полосы $v_{3}$ смещается на $14 \mathrm{~cm}^{-1}$ в длинноволновую, а положение полосы $v_{4}-$ на $3 \mathrm{~cm}^{-1}$ в коротковолновую область спектра. Положения максимумов полос отражения $v_{3}$ и $v_{4}$ в $Y$-направлении кристалла $\left(\mathrm{NH}_{4}\right)_{2} \mathrm{SO}_{4}$ смещаются соответственно на 4 и $3 \mathrm{~cm}^{-1}$ в коротковолновую область, тогда как для кристалла $\mathrm{LiNH}_{4} \mathrm{SO}_{4}$ положение полосы $v_{3}$ смещается соответственно на 23 и $1 \mathrm{~cm}^{-1}$ в длинноволновую область спектра. В $Z$-направлении кристалла $\left(\mathrm{NH}_{4}\right)_{2} \mathrm{SO}_{4}$ полосы $v_{3}$ и $v_{4}$ смещаются соответственно на 3 и $4 \mathrm{~cm}^{-1}$ в коротковолновую область, а для кристалла $\mathrm{LiNH}_{4} \mathrm{SO}_{4}$ полоса $v_{3}$ смещается на $17 \mathrm{~cm}^{-1}$ в длинноволновую, а полоса $v_{4}-$ на $6 \mathrm{~cm}^{-1}$ в коротковолновую область спектра.

Кроме изменения положения максимумов при одноосном сжатии кристалла $\mathrm{LiNH}_{4} \mathrm{SO}_{4}$ обнаружено также изменение интенсивности отраженных лучей. Так, для спектров отражения в $X$-направлении интенсивность увеличивалась для значения одноосного сжатия $16 \mathrm{bar}$ вдоль оси $Z$, а потом при увеличении давления от 32 до 80 bar резко уменьшалась по сравнению со свободным кристаллом. Интенсивность полосы $v_{3}$ в $Y$-направлении кристалла $\mathrm{LiNH}_{4} \mathrm{SO}_{4}$ при комнатной температуре при значении одноосного сжатия вдоль оси $X 42$ bar увеличивалась по сравнению со свободным кристаллом, а при 63 и 84 bar резко уменьшалась, тогда как интенсивность полосы $v_{4}$ увеличивалась для всех значений одноосного сжатия. Для $Z$-направления кристалла $\mathrm{LiNH}_{4} \mathrm{SO}_{4}$ при комнатной температуре при одноосном сжатии вдоль оси $Y$ до 34 bar интенсивность отраженных лучей увеличилась по сравнению с интенсивностью отражения свободного образца, далее она уменьшалась, а при $\sigma \sim 84$ bar - снова увеличивалась.

В общем наблюдается неоднозначное поведение коэффициента поглощения кристалла $\mathrm{LiNH}_{4} \mathrm{SO}_{4}$ для разных значений одноосного сжатия.

С помощью дисперсионных соотношений КрамерсаКронига за спектрами отражений получены барические изменения спектральных зависимостей оптических постоянных: показателя преломления $n$, действительной $\varepsilon_{1}$ и мнимой $\varepsilon_{2}$ частей диэлектрической проницаемости кристалла СА вдоль трех кристаллофизических осей. Обнаружено резкое увеличение показателя преломления в области полос поглощения, а именно $\partial n / \partial \lambda \sim 4.52$

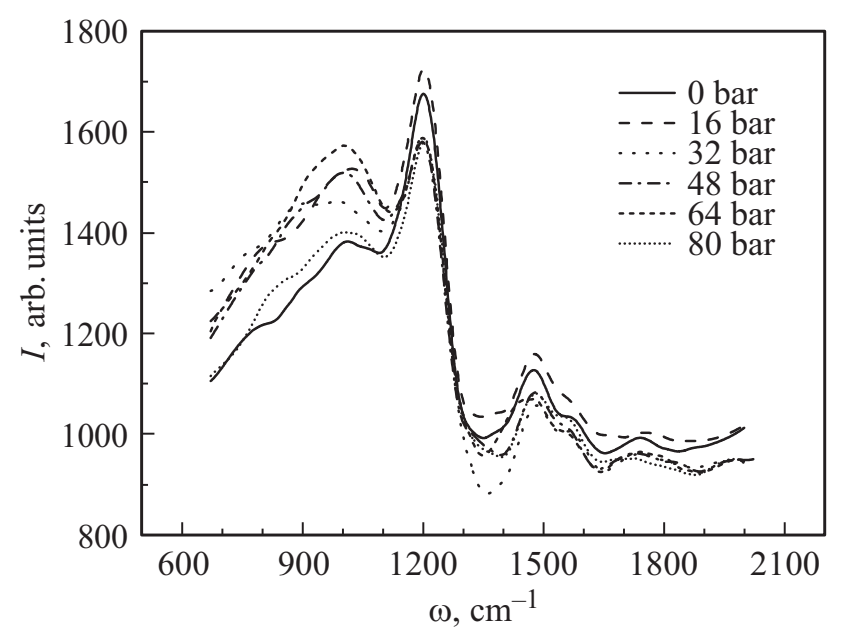

Рис. 2. ИК спектры отражения в $X$-направлении кристалла $\mathrm{LiNH}_{4} \mathrm{SO}_{4}$ при комнатной температуре для разных значений одноосного сжатия вдоль оси $Z$.

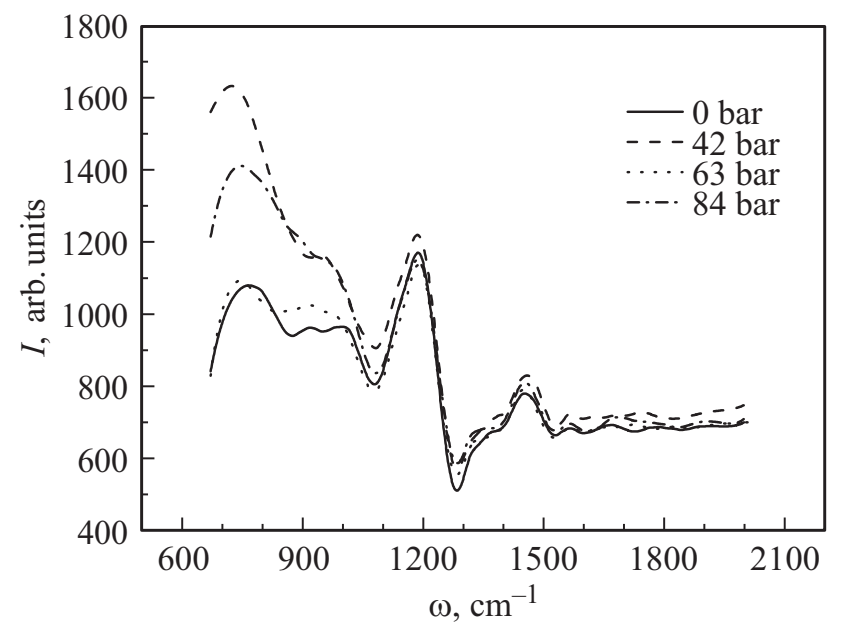

Рис. 3. ИК спектры отражения в $Y$-направлении кристалла $\mathrm{LiNH}_{4} \mathrm{SO}_{4}$ при комнатной температуре для разных значений одноосного сжатия вдоль оси $X$.

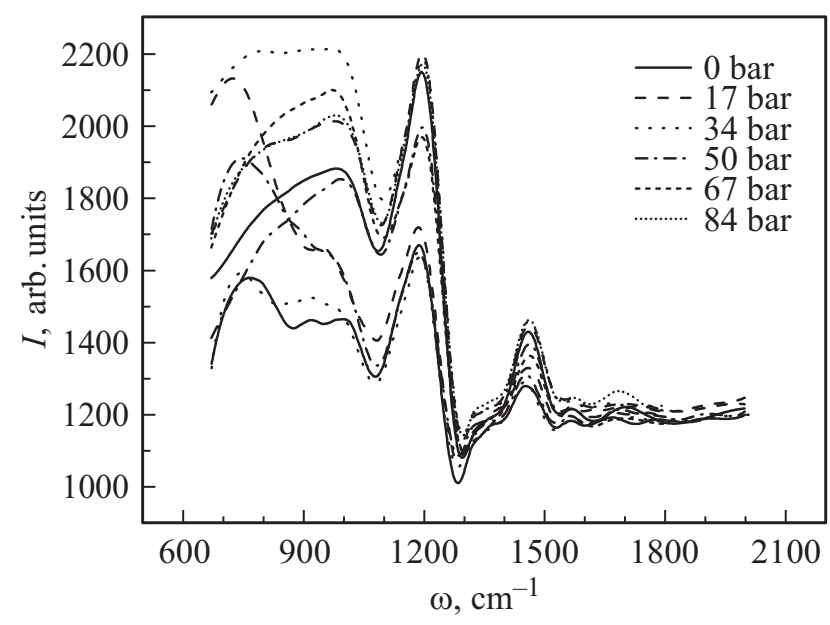

Рис. 4. ИК спектры отражения в Z-направлении кристалла $\mathrm{LiNH}_{4} \mathrm{SO}_{4}$ при комнатной температуре для разных значений одноосного сжатия вдоль оси $Y$. 

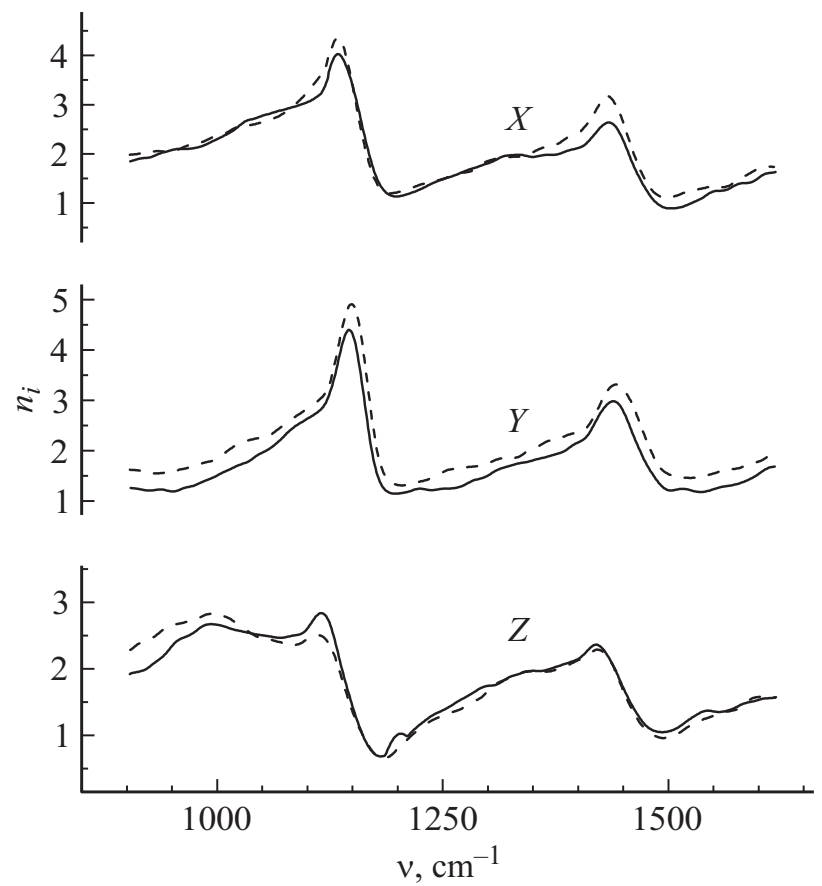

Рис. 5. Спектральная зависимость показателя преломления $n_{i}$ кристалла $\mathrm{LiNH}_{4} \mathrm{SO}_{4}$ при комнатной температуре для разных поляризаций и разных направлений давлений $\sigma_{m}=100 \mathrm{bar}$, сплошная линия - механически свободный, штриховая зажатый образец.

(полоса $v_{3}$ ) и $2.47 \mu \mathrm{m}^{-1}$ (полоса $v_{4}$ ) (рис. 5). Изменение показателя преломления вдали полосы поглощения соответствует нормальной дисперсии $\partial n / \partial \lambda<0$. Сравнение величины нормальной дисперсии в данных областях с дисперсией в видимой части спектра свидетельствует об их приблизительном равенстве. Так, например, $\partial n / \partial \lambda \sim 0.012 \mu \mathrm{m}^{-1}$ в спектральной области $1100-1200 \mathrm{~cm}^{-1}$, тогда как $\partial n / \partial \lambda \sim 0.01 \mu \mathrm{m}^{-1}$ в видимой части спектра (последнее значение получено в результате измерения показателя преломления интерференционным методом Обреимова).

Как видно из рис. 5, в направлениях $X$ и $Y$ показатель преломления существенно увеличивается $\delta n \sim 0.55-0.78$, тогда как в направлении $Z$ он уменьшается. Характер изменений показателей преломления в ИК области воссоздает характер поведения $n$ в видимой части спектра. Ранее [12-14] для большинства кристаллов типа $\mathrm{A}_{2} \mathrm{BX}_{4}\left(\mathrm{LiKSO}_{4}, \mathrm{LiRbSO}_{4}, \mathrm{RbNH}_{4} \mathrm{SO}_{4}\right.$, $\left.\left(\mathrm{NH}_{4}\right)_{2} \mathrm{BeF}_{4}\right)$ установлено барическое увеличение показателей преломления $\delta n \sim 10^{-2}-10^{-3}$, что обусловлено увеличением в первую очередь плотности частиц кристалла. Если исходить из известной формулы ЛорентцЛоренца:

$$
\frac{n^{2}-1}{n^{2}+2}=\frac{4}{3} \pi N \alpha
$$

$(n-$ показатель преломления, $\alpha-$ электронная поляризуемость, $N$ - число частиц в единице объема), то видно, что увеличение показателя преломления, обу- словленное одноосным сжатием, ведет к увеличению $\alpha_{i}$ кристалла. Из графика также видно незначительное барическое смещение пиков, что соответствует барическому смещению максимумов полос в ИК спектрах отражения.

Исходя из рассчитанных дисперсионных кривых действительной $\varepsilon_{1}$ части диэлектрической проницаемости, были определены частоты продольных колебаний $v_{\mathrm{LO}}$ как минимумы функции $\varepsilon_{1}$ и частоты поперечных колебаний $\varepsilon_{\mathrm{TO}}$ как максимумы функции $\varepsilon_{2}$ для механически свободных и одноосно зажатых кристаллов (табл. 2).

Константа затухания $\gamma$ определялась как полуширина соответствующего максимума кривой $\varepsilon_{2}$, а сила осциллятора определялась как величина

$$
f \sim n^{2}\left(v_{\mathrm{LO}}-v_{\mathrm{TO}}\right),
$$

где $n$ - показатель преломления с высокочастотной стороны от соответствующей полосы.

Как видно из табл. 2, одноосные давления $\sigma_{x}$ и $\sigma_{y}$ ведут к уменьшению частот продольных и поперечных колебаний в обеих полосах отражения, тогда как давления $\sigma_{z}$ смещают эти частоты в сторону более высоких энергий. Кроме того, одноосные давления вдоль трех кристаллофизических направлений уменьшают константу затухания и силу осциллятора полосы III и увеличивают силу осциллятора полосы II.

Полученные результаты обусловлены влиянием одноосных напряжений на структуры данного кристалла. При комнатной температуре структура кристалла является разупорядоченной по отношению к ориентации тетраэдрических $T$-групп, которые совершают либрационные колебания большой амплитуды вокруг псевдогексагональной оси $Z$ и оси $Y$. Базовая ячейка кристалла $\mathrm{LiNH}_{4} \mathrm{SO}_{4}$ содержит два псевдогексагональных каркаса с тетраэдрами $\mathrm{SO}_{4}$ и $\mathrm{NH}_{4}$, которые развернуты вдоль разных кристаллофизических осей. Приложение одноосных давлений вдоль разных осей будет тормозить или ускорять повороты $T$-групп, тем самым несколько деформировать элементарную ячейку. Это проявляется в уменьшении или увеличении частот продольных и поперечных колебаний под давлением. Наблюдаемое барическое

Таблица 2. Частоты продольных $v_{\text {LO }}$ и поперечных $v_{\text {то }}\left(\mathrm{cm}^{-1}\right)$ колебаний, константа затухания $\gamma$ и сила осциллятора $f$ механически свободного и зажатого $\left(\sigma_{m}=100 \mathrm{bar}\right)$ кристалла $\mathrm{LiNH}_{4} \mathrm{SO}_{4}$

\begin{tabular}{c|c|c|c|c|c|c|r|r}
\hline \multirow{2}{*}{ Направление } & \multicolumn{2}{|c|}{$v_{\mathrm{LO}}$} & \multicolumn{2}{c|}{$v_{\mathrm{TO}}$} & \multicolumn{2}{c|}{$\gamma$} & \multicolumn{2}{c}{$f$} \\
\cline { 2 - 9 } & $\sigma=0$ & $\sigma$ & $\sigma=0$ & $\sigma$ & $\sigma=0$ & $\sigma$ & $\sigma=0$ & $\sigma$ \\
\hline$E \| X$ & 1214 & 1201 & 1184 & 1172 & 42 & 34 & 118 & 115 \\
& 1476 & 1479 & 1454 & 1449 & 66 & 67 & 65 & 51 \\
\hline$E \| Y$ & 1210 & 1187 & 1192 & 1170 & 25 & 21 & 114 & 115 \\
& 1455 & 1454 & 1430 & 1428 & 79 & 71 & 79 & 69 \\
\hline$E \| Z$ & 1213 & 1216 & 1184 & 1187 & 38 & 33 & 101 & 104 \\
& 1456 & 1460 & 1428 & 1434 & 62 & 59 & 75 & 69
\end{tabular}


уменьшение сил осциллятора соответствующих полос свидетельствует об уменьшении либрационных колебаний великой амплитуды как тетраэдров $\mathrm{SO}_{4}$, так и $\mathrm{NH}_{4}$.

Поскольку несколько большие как по интенсивности, так и по частоте барические изменения обнаружены для полосы $v_{3}$, которая ответственна за колебания тетраэдра $\mathrm{SO}_{4}$, то можно предположить, что эти колебания в основном определяют физические свойства данного кристалла, хотя не стоит преуменьшать и роль колебаний тетраэдров $\mathrm{NH}_{4}$-групп.

Таким образом, впервые исследованы ИК спектры отражения механически свободного и зажатого одноосными давлениями кристалла ЛАС в спектральной области 600-2000 $\mathrm{cm}^{-1}$ вдоль трех кристаллофизических направлений. С помощью дисперсионных соотношений Крамерса-Кронига получены дисперсионные и барические зависимости показателя преломления $n$, а также рассчитаны параметры, характеризирующие ИК дисперсию: частоты продольных $\omega_{\mathrm{LO}^{-}}$и поперечных

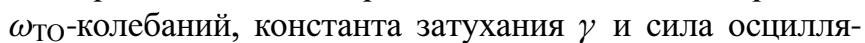
тора $f$ механически свободного и зажатого одноосными давлениями кристалла $\mathrm{LiNH}_{4} \mathrm{SO}_{4}$. Обнаружены значительные барические изменения как интенсивности, так и частоты главных полос отражения, что объясняется влиянием одноосных давлений на структуру кристалла, а именно на тетраэдрические остовы $\mathrm{NH}_{4}$ и $\mathrm{SO}_{4}$.

Публикация содержит результаты исследований, проведенных при грантовой поддержке Государственного фонда фундаментальных исследований за конкурсной работой № Ф73/101-2016.

\section{Список литературы}

[1] Groth P. Chemische Kristallographie. Leizpig, 1908. 367 p.

[2] Potska M. // Phase Transitions: A Multinational J. 2001. V. 12. P. 409.

[3] Pietraszko A., Lukaszewicz K. // Pol. J. Chem. 1992. N 66. P. 2057.

[4] Nakamura T., Kojima S., Takashige M. // Jpn. J. Appl. Phys. 1979. N 18. P. 711.

[5] Tomaszewski P.E. // Solid State Commun. 1992. N 81. P. 333.

[6] Рудиш М.Я., Стадник В.И., Брезвин Р.С., Щепанский П.А. // ФТТ. 2015. Т. 57. № 1. С. 50-55.

[7] Stadnyk V.Y., Brezvin R.S., Savchuk P.V. // Opt. and Spectrosc. 2012. V. 113. N 3. P. 28.

[8] Alam S., Srivastava J.P. // Spectrochim. Acta A. 1981. N 37. P. 183.

[9] Torgashev V.I., Yuzyuk Y.I., Smutny F. // Phys. Stat. Sol. B. 1986. N 135. P. 93.

[10] Sosnowska I., Hilczer B., Pskunowicz P. // Solid State Commun. 1990. N 74. P. 1249.

[11] Polonska M., Hilczer B., Baran J.// J. Mol. Struct. 1994. N 325. P. 105.

[12] Stadnyk V.Yo., Gaba V.M., Andrievski B.V., Kohut Z.O. // Phys. of the Solid States. 2011. V. 53. N 1. P. 131.

[13] Stadnyk V.J., Romanyuk M.O. // Phys. Stat. Sol. (A). 1996. V. 158. P. 289.
[14] Andriyevsky B., Romanyuk M., Stadnyk V. // J. Phys. and Chemistry of Solids. 2009. V. 70. P. 1109.

[15] Komornicka D., Wotcyrz M., Pietraszko A. // Sol. Stat. Chem. 2015. N 230. P. 325.

[16] Stadnyk V.Yo., Romanyuk M.O., Tuzyak N.R. // Phys. Sol. Stat. 2007. V. 49. N 4. P. 696. 